\title{
Performed Lesion Description Lesion Number
}

National Cancer Institute

\section{Source}

National Cancer Institute. Performed Lesion Description Lesion Number. NCI Thesaurus.

Code C93926.

The integer assigned to a lesion for a subject. 